\title{
IN MEMORIAM EMMANUEL BELIN
}

\begin{abstract}
Emmanuel Belin était aspirant du Fonds National de la Recherche Scientifique et chercheur au Département de communication. Né à Rocourt le 24 octobre 1968, il est décédé dans des circonstances tragiques le 18 janvier 1998.
\end{abstract}

Emmanuel était un être fort et fragile à la fois. Un être complexe aussi. Trop peut-être, pour espérer vivre simplement, pour s'imprégner de la vie... sans penser. Il ne semblait pas pouvoir échapper à ce besoin de se poser les questions jusqu'où elles ne se posent pas, nous renvoyant inévitablement à une part inaccessible de nous-mêmes.

Chercheur de génie, il était pour les autres d'une grande disponibilité trouvant toujours le moyen de les faire progresser dans leur réflexion. La désinvolture soignée qu'il affichait avait le don de faire sourire ou d'émouvoir les étudiants et les collègues qu'il côtoyait. Mais sa prise de parole, l'affinement de ses jugements, sa capacité d'interprétation personnelle en faisait un être rigoureux, exigeant et engagé.

Car il était aussi acteur de la cité, comme en témoigne son engagement dans les "cafés politiques". Du reste, il était attentif à toujours trouver quelque chose de valorisant dans toutes les formes de l'expérience des "gens ordinaires", y compris celle des fans de soap opera ou de celles qu'il appelait "mes jeunes femmes précaires".

Emmanuel avait un sens profond des circonstances de l'existence, de l'ennui et de l'anxiété qui la traversent. Il a su le traduire dans une écriture rigoureuse dont la puissance poétique ne pouvait que renforcer le caractère habité de sa réflexion. Il a réussi à 
thématiser ce qui le tourmentait pour le transformer en une élucidation lumineuse d'un aspect essentiel, bien que peu visible, des dispositifs techniques contemporains: leur bienveillance, leur caractère de monde intermédiaire, bref leur disposition à rendre le monde habitable. De manière plus large, il avait, à partir de l'idée d'objet transitionnel de Winnicott, développé une sociologie des espaces potentiels dont on pressent la fécondité heuristique pour la compréhension de toutes les "machines à communiquer".

Cela ne l'a pas aidé à vivre, mais le travail qu'il nous laisse nous reste comme une "œuvre" qui rend la vie plus intelligible et qu'il importe, pour cela même, d'approfondir" .

1 On trouvera ci-après une de ses ultimes réflexions. 